\title{
Effect of Sleep on Regional Blood Flow Distribution in Piglets
}

\author{
AURORE COTE AND GABRIEL G. HADDAD
}

Department of Pediatrics (Respiratory Medicine Service), Montreal Children's Hospital-Research Institute, McGill University, Montreal, Canada H3H 1 P3 and Department of Pediatrics (Section of Respiratory Medicine), Yale University School of Medicine, New Haven, Connecticut 06510

\begin{abstract}
The regional distribution of blood flow to the brain and to other major organs was studied during wakefulness and sleep in growing piglets. A young group was studied at $6.8 \pm 1.3 \mathrm{~d}$ of age and an older group at $33.5 \pm 5.5 \mathrm{~d}$. Two $\mathrm{d}$ before the experiments, we instrumented the animals for measurement of blood flow by the microsphere technique. We determined sleep state using EEG and behavioral criteria. Although we did not find significant differences in blood gas tensions and cardiac output with changes in behavioral states, we did note a number of important changes in brain and muscle blood flow with sleep. 1) Although total brain blood flow changed little between wakefulness and sleep at both ages, regional differences existed. Indeed, at both ages, during rapid eye movement sleep (active sleep), blood flow to the thalamushypothalamus and brainstem was significantly higher than during wakefulness $(p<0.025)$; in older piglets, blood flow to these two regions was significantly lower in quiet sleep than in wakefulness $(p<0.05) .2)$ Blood flow to most skeletal muscle groups, and particularly to the diaphragm, was lower during sleep than during wakefulness. 3) Age did not have a significant effect on the regional distribution of blood flow during sleep. We conclude that behavioral states influence the regional distribution of blood flow in early life, but not in an age-dependent fashion. We speculate that, because no difference was observed in other hemodynamic variables, the regional changes in organ blood flow with sleep most probably reflect the differences in local metabolic needs. (Pediatr Res 28: 218-222, 1990)
\end{abstract}

Behavioral states are known to influence cardiovascular function in a major way in both animals and humans (1). For example, there is a decrease in heart rate, blood pressure, and cardiac output when passing from wakefulness to quiet sleep; further decreases in blood pressure and heart rate also occur during tonic rapid eye movement sleep (or active sleep) and marked heart rate variability and blood pressure fluctuations characterize the phasic active sleep period (1). Behavioral states are also known to influence the distribution of cardiac output. For example, Mancia et al. (2), using flow probes placed around several vessels in adult cats, have shown a redistribution of cardiac output in active sleep accompanied by vasodilatation in skin and mesenteric and renal vascular beds, and vasoconstriction in the skeletal muscular vascular bed (2). Other investigators

Received February 12, 1990; accepted April 26, 1990.

Reprint requests: Aurore Cote, Respiratory Medicine Service, Montreal Children's Hospital, 2300 rue Tupper, Montreal, Oc, Canada H3H 1 P3.

Supported by National Heart, Lung and Blood Institutes, Grants HD-14565 and HD-15736. This work was performed during the tenure by G.G.H. of an Established Investigatorship of the American Heart Association. A.C. was a Fellow of the Fonds de la Recherche en Sante du Quebec. have noted increases in brain blood flow during active sleep as compared with wakefulness and quiet sleep in both humans and animals (3-5).

Although the influence of behavioral states on heart rate, blood pressure, and cardiac output has been studied, there is, to our knowledge, virtually no information about the influence of behavioral states on regional distribution of blood flow in early life $(6,7)$. We therefore asked $l$ ) how the various states of consciousness affect organ blood flow in the young and, more specifically, 2) how blood flow within the different organ regions (for example within the brain) varies from one behavioral state to another. We accordingly performed experiments in young and older piglets, studying blood flows to organ subregions in three separate states: active sleep, quiet sleep, and wakefulness. Piglets were chosen because the maturation of their autonomic nervous system and cardiovascular function, as well as their brain development, occurs postnatally in the first few weeks of life $(8,9)$ and correlates well with human postnatal development.

\section{MATERIALS AND METHODS}

Animal preparation. The animals used for these studies were divided into two groups: young piglets [mean age $6.8 \pm 1.3 \mathrm{~d}$ (SD); mean wt $2.0 \pm 0.5 \mathrm{~kg}$ ] and older piglets (mean age $33.5 \pm$ $5.5 \mathrm{~d}$ and mean wt $7.7 \pm 1.9 \mathrm{~kg}$ ). The animals were obtained a few days before the experiments and kept in our animal quarters. We provided the young piglets with sow milk replacer ad libidum throughout day and night and the older ones with a combination of sow milk replacer and solid food. The environmental temperature in the animal quarters was kept between 26 and $30^{\circ} \mathrm{C}$, although additional heat was provided for the young piglets (32$\left.33^{\circ} \mathrm{C}\right)(10,11)$.

We performed surgery for catheter insertion 2 to $3 \mathrm{~d}$ before the experiments, using halothane $/ \mathrm{N}_{2} \mathrm{O} / \mathrm{O}_{2}$ anesthesia. In young piglets, one catheter was inserted in the left atrium from a femoral vein via the foramen ovale ( 5 Fr polyurethane, NIH type; Cordis Laboratories, Inc., Miami, FL), and a second in the descending aorta from a femoral artery (polyvinyl, Tygon microbore tubing, Norton Co., Wayne, NJ). In older piglets, one catheter was inserted in the left ventricle ( 4 or 5 Fr polyethylene, pediatric pigtail type, Cook Co., Bloomingdale, IN) and a second in the descending aorta (polyvinyl). In older piglets, the left ventricle was used as the site of injection of microspheres inasmuch as the foramen ovale is rarely patent at that age. The aortic catheter was positioned below the renal arteries. The position of all catheters was verified by fluoroscopy. Once the anesthesia was stopped, the animals woke up rather rapidly (15-20 min); within a few hours after surgery, they appeared to behave, walk, and feed normally.

Blood flow measurement. We measured blood flow using the microsphere technique described by Heymann et al. (12). The microspheres were $15 \pm 5 \mu \mathrm{m}$ in diameter and were labeled with one of the following radionuclides: ${ }^{46} \mathrm{Sc},{ }^{95} \mathrm{Nb},{ }^{103} \mathrm{Ru},{ }^{113} \mathrm{Sn}$, or 
${ }^{57} \mathrm{Co}$ (New England Nuclear, Boston, MA); we injected $9 \times 10^{5}$ microspheres into the young and $1.5 \times 10^{6}$ microspheres into the older piglets. These microspheres were injected into the left heart over a period of $30 \mathrm{~s}$, and we withdrew a reference sample from the descending aorta at a rate of $2 \mathrm{~mL} / \mathrm{min}$ in the young and $4 \mathrm{~mL} / \mathrm{min}$ in the older piglets, for a total of $2 \mathrm{~min}$. Blood withdrawal started $15 \mathrm{~s}$ before the injection of microspheres. All blood losses were replaced with blood from a compatible piglet donor immediately after the measurement of blood flow.

At the end of the experiment, we killed the animals with an overdose of pentobarbital. The brain was then removed and the following regions were identified and separated: cerebral hemispheres, cerebellum, thalamus-hypothalamus, and brainstem. The brainstem was further divided into midbrain, pons, and medulla oblongata. We assessed the thalamus and hypothalamus together because no clear anatomical division separated the two. The heart, adrenals, kidneys, spleen, and diaphragm were taken as whole organs. We further separated the heart into right and left ventricles (free wall only) and took samples in a systematic way from the liver, gastrointestinal tract, intercostals and skeletal muscles, and the skin. The samples from the skeletal muscles were taken from the fore limb (unaffected by arterial catheterization), and the skin samples included s.c. tissue. Each tissue sample from the various organs was counted in a well-type gamma counter (Packard Instrument Co., Inc., Downers Grove, IL). We used a computer program to correct the individual counts for spillover of the different radionuclides and to calculate the blood flow (13).

Validation of microsphere technique in piglets. The microsphere technique has been validated in acutely instrumented, awake or anesthetized piglets. Because we used unanesthetized animals during both wakefulness and sleep at two different ages and with different microsphere injection sites, we had to validate the technique in our piglet preparation. In a series of experiments $(n=6)$, we therefore examined the degree of evenness of mixing in the circulation, the adequacy of the site of withdrawal for the reference sample, the optimum withdrawal rate, the adequate dose of microspheres, and the absence of hemodynamic changes with the repeated injections. In all the studies reported, we also tested the evenness of the microsphere mixing in the circulation by comparing the blood flow to paired organs such as kidneys and cerebral hemispheres. We could detect no evidence of unevenness of mixing.

Cardiac output measurement. We measured cardiac output using the dye-dilution technique. To that end, we injected Cardiogreen (Hynson, Westcott, and Dunning, Inc., Baltimore, MD) into either the left atrium (young piglets) or the inferior vena cava (older piglets) and withdrew blood from the arterial catheter, passing it through a calibrated densitometer. The doses of Cardiogreen ranged from 0.5 to $0.75 \mathrm{mg}$ for the young and from 0.75 to $1.25 \mathrm{mg}$ for the older piglets.

The technique was also used to verify that no shunting existed between the aorta and the pulmonary artery through the ductus arteriosus.

Determination of behavioral states. We used both EEG and behavioral criteria to determine the state of consciousness. The EEG was recorded via thin s.c. stainless steel wires inserted over the temporoparietal region (14) and the following criteria were used to differentiate the three states: wakefulness was characterized as low-amplitude high-frequency waves on the EEG, eyes open, and no movement (although there were periods during wakefulness that were characterized by moderate activity, we considered only those periods during which the piglets were calm and lying down in the study chamber); quiet sleep involved eyes closed, no movement, and high-amplitude slow waves on the EEG; active sleep involved eyes closed, frequent rapid eye movements, multiple twitching of the muscles of the face and of the extremities, and low-amplitude high-frequency waves on the EEG. We made no attempt to differentiate tonic from phasic active sleep.
Experimental protocol. We studied the animals in a Plexiglass chamber in which the temperature was maintained in the thermoneutral range, that is, at a temperature between 32 and $33^{\circ} \mathrm{C}$ for the young piglets and between 26 and $30^{\circ} \mathrm{C}$ for the older ones $(10,11)$. A gas circulation system was used to control $\mathrm{CO}_{2}, \mathrm{O}_{2}$, and $\mathrm{H}_{2} \mathrm{O}$ vapor. $\mathrm{CO}_{2}$ was kept below $0.1 \%, \mathrm{O}_{2}$ above $19.8 \%$, and $\mathrm{H}_{2} \mathrm{O}$ between 50 and $70 \%$.

The animals were not sleep deprived before the study and were fed normally on the morning of the experiment. During each experiment, the animals could move freely and were allowed to stay awake or fall asleep spontaneously. The studies started at about $1000 \mathrm{~h}$ and usually lasted 2 to $3 \mathrm{~h}$. We conducted 5 to 10 min of baseline recordings in each behavioral state at the beginning of each experiment. After a specific state had been clearly identified and had lasted for at least $30 \mathrm{~s}$, we then administered the various microsphere injections (the order of the isotope having been chosen randomly). Because there could be variability in blood flow to organs within a single state, we performed two measurements in each state. If the animal changed state during the $2 \mathrm{~min}$ required to collect the reference sample, the trial was

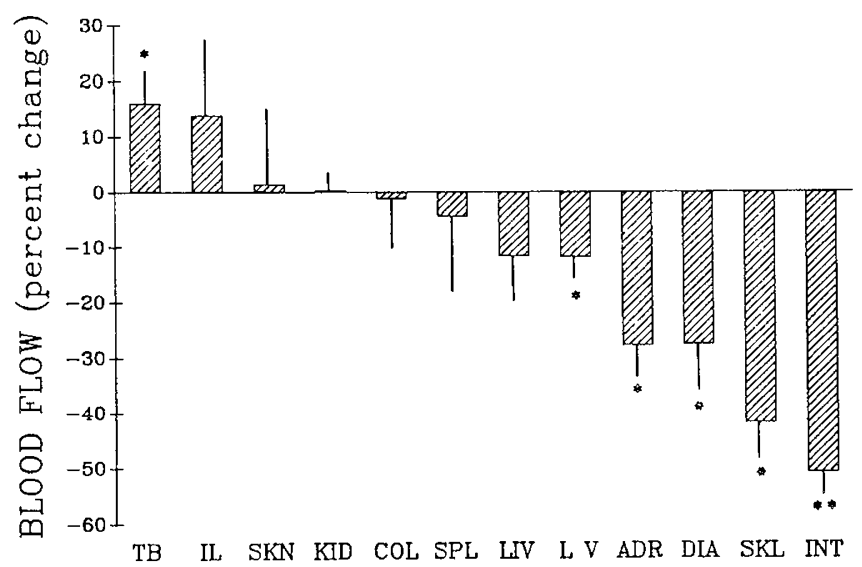

Fig. 1. Influence of active sleep on regional distribution of blood flow. The results are expressed as \% change in blood flow (mean \pm SE) between active sleep and wakefulness, wakefulness considered as baseline. $T B$, total brain; $I L$, ileum; $S K N$, skin; KID, kidneys; $C O L$, colon; $S P L$, spleen; $L I V$, liver; $L V$, left ventricle; $A D R$, adrenals; $D I A$, diaphragm; $S K L$, skeletal muscles; $I N T$, intercostals. Significance based on difference in blood flow between the two states; statistical analysis done with the values of blood flow, not the \% difference. ${ }^{*} p<0.05,{ }^{* *} p<0.01$.

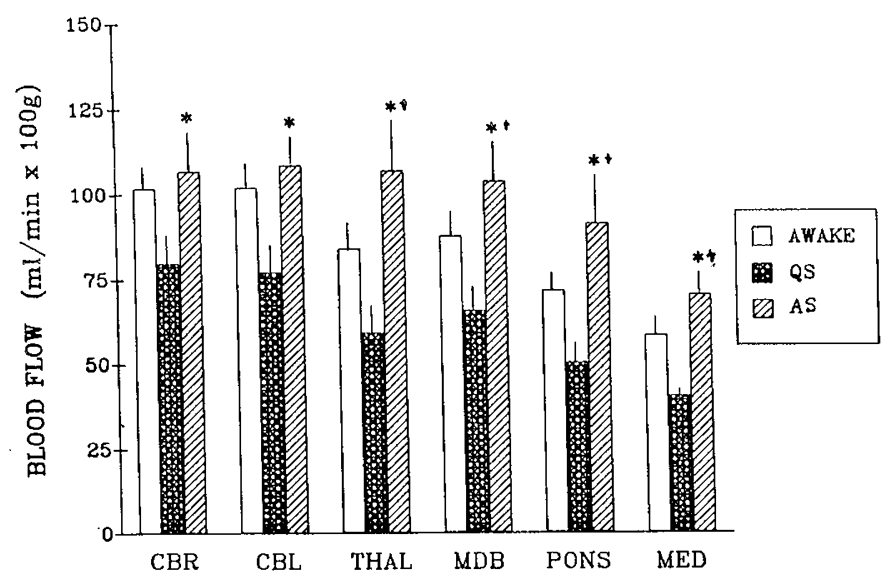

Fig. 2. Regional brain blood flow during wakefulness, quiet sleep $(Q S)$, and active sleep $(A S)$ in older piglets. Note that blood flow to the thalamus-hypothalamus and some regions of the brainstem was significantly higher in active sleep than during quiet sleep and wakefulness. Blood flow is expressed in $\mathrm{mL} / \mathrm{min} \times 100 \mathrm{~g}$; values are mean $\pm \mathrm{SE} .{ }^{*} p$ $<0.005 \mathrm{AS} v s \mathrm{QS}, \dagger p<0.05 \mathrm{AS} v s$ wakefulness. 
rejected. At the end of this 2 -min period, we withdrew an arterial blood sample for analysis of blood gas tensions. In addition, we measured the hematocrit at the beginning and end of the study.

Our protocol was approved by the Animal Care Committee of the institution.

Data analysis. Although we were able to study all three states in older piglets, we could not examine blood flow in quiet sleep in the young. Instead, quiet sleep occurs relatively infrequently in young piglets and when it is present, it is generally of a very short duration (less than $2 \mathrm{~min}$ ). We therefore studied only active sleep and wakefulness in the young.

We averaged the different measurements of blood flow in the same state for each animal and then calculated the mean value $\pm \mathrm{SD}$ for the group. To compare the results between states within the same age group, we used either the paired $t$ test when only two states were compared or a single-factor analysis of variance when a comparison of repeated measures was necessary. Comparisons with a significant $F$ value were then further evaluated with the Newman-Keuls' multiple range test. To compare the two age groups, we used a two-way analysis of variance with age as the between-group factor and state as the within-group factor (15). Differences in means were considered statistically significant if $p<0.05$.

\section{RESULTS}

The regional distribution of blood flow during sleep was studied in a total of 20 piglets. We succeeded in studying all three states in six older piglets. In the other animals of that age group $(n=7)$, only wakefulness and active sleep could be examined, thus giving us a total of 13 older piglets that we could compare with seven young piglets in terms of wakefulness and active sleep. In all studies, we had very good reproducibility of the microsphere measurement of blood flow in the same animal during wakefulness (at both ages) and during quiet sleep (older animals).

On the basis of our results vis-à-vis regional distribution of blood flow, we were able to divide the different organs studied into three categories. This categorization was especially clear when we compared wakefulness and active sleep (Fig. 1). We found that the brain regions such as thalamus-hypothalamus and brainstem had a significantly higher blood flow during active sleep than during quiet sleep or wakefulness. The muscle groups such as skeletal muscles and diaphragm had, in general, a lower blood flow during sleep than during wakefulness. Finally, organs such as the spleen, liver, gastrointestinal tract, and the skin did not exhibit a preferential change in blood flow as a function of state.

Effect of Behavioral States on Regional Distribution of Blood Flow. Regional brain blood flow. The six older piglets in which measurements were obtained in all three states clearly demonstrated a significant effect of state in both the brainstem and the thalamus-hypothalamus. Figure 2 shows that these regions had a higher rate of blood flow during active sleep than during wakefulness or quiet sleep, the latter state having the lowest value. Indeed, the average percent difference in blood flow between active and quiet sleep was quite marked for the thalamus-hypothalamus $(81 \%)$ and all regions of the brainstem $(60-$ $82 \%$ ).

No significant effect of age appeared when the effects of active sleep were compared in the young and the older piglets. However, Table 1 shows that the young piglets had a mean total brain blood flow during active sleep that was significantly higher than during wakefulness. The older piglets had a mean total brain blood flow during wakefulness that was not significantly different from that in active sleep. In fact, of the 13 older animals studied, six had a higher total brain blood flow in active sleep, three had a lower value in active sleep, and four showed little change. There was, however, a preferential distribution of blood flow to the thalamus-hypothalamus and brainstem in active sleep at both ages. This preferential distribution of blood flow was seen in all animals, including those older piglets with a lower total brain blood flow in active sleep than in wakefulness.

Heart and skeletal muscle blood flow. Sleep was found to have a significant effect on muscle blood flow. In older piglets, during both active and quiet sleep, blood flow to the left ventricle, diaphragm, and intercostal muscles was significantly lower than during periods of wakefulness (Table 2). No significant difference was found, however, between the two sleep states. Also, blood flow to the skeletal muscles was not influenced by sleep.

Table 1 shows the comparison of blood flow to the heart and skeletal muscles during wakefulness and active sleep between the young and the older piglets. Clearly, the influence of sleep on muscle blood flow was not age dependent.

Other organs. Behavioral state did not have any effect on the blood flow to kidneys, spleen, liver, gastrointestinal tract, and skin. Blood flow to the adrenals, however, was higher during wakefulness than during active sleep at both ages.

Cardiac output, hemodynamic status. Cardiac output was measured in a subgroup of six young and six older piglets in the different behavioral states because we wanted to know whether a change in cardiac output was responsible for the changes

Table 1. Regional blood flow during wakefulness and active sleep*

\begin{tabular}{|c|c|c|c|c|}
\hline & \multicolumn{2}{|c|}{ Young } & \multicolumn{2}{|c|}{ Older } \\
\hline & Wakefulness & Active sleep & Wakefulness & Active sleep \\
\hline Total brain & $94 \pm 8$ & $111 \pm 19 \dagger$ & $97 \pm 18$ & $107 \pm 21$ \\
\hline Cerebrum & $94 \pm 8$ & $109 \pm 19 \dagger$ & $100 \pm 19$ & $107 \pm 22$ \\
\hline Cerebellum & $96 \pm 14$ & $110 \pm 18$ & $98 \pm 19$ & $110 \pm 18$ \\
\hline Thalamus-hypothalamus & $95 \pm 14$ & $129 \pm 33 \dagger$ & $91 \pm 28$ & $119 \pm 38 \ddagger$ \\
\hline Brainstem & $86 \pm 10$ & $110 \pm 24 \dagger$ & $73 \pm 13$ & $92 \pm 22 \ddagger$ \\
\hline Midbrain & $96 \pm 9$ & $127 \pm 26 \ddagger$ & $89 \pm 18$ & $109 \pm 27 \dagger$ \\
\hline Pons & $86 \pm 10$ & $110 \pm 24 \dagger$ & $69 \pm 19$ & $92 \pm 29 \ddagger$ \\
\hline Medulla & $74 \pm 5$ & $94 \pm 17 \dagger$ & $58 \pm 11$ & $76 \pm 16 \ddagger$ \\
\hline RV & $150 \pm 27$ & $136 \pm 20$ & $155 \pm 46$ & $158 \pm 57$ \\
\hline LV & $283 \pm 26$ & $249 \pm 37 \dagger$ & $236 \pm 47$ & $208 \pm 35 \dagger$ \\
\hline Diaphragm & $42 \pm 12$ & $29 \pm 6 \dagger$ & $40 \pm 17$ & $27 \pm 10 \dagger$ \\
\hline Intercostals & $27 \pm 11$ & $13 \pm 7 \ddagger$ & $18 \pm 9$ & $12 \pm 9$ \\
\hline Skeletal muscles & $20 \pm 9$ & $11 \pm 3 \dagger$ & $16 \pm 8$ & $13 \pm 9$ \\
\hline
\end{tabular}

$*$ Results expressed in $\mathrm{mL} / \mathrm{min} \times 100 \mathrm{~g}$. Values are mean $\pm \mathrm{SD}$. Young, $n=7$; Older, $n=13$. Significance based on paired data. $\mathrm{RV}$, right ventricle; $L V$, left ventricle.

$\dagger p<0.05$.

$\ddagger p<0.01$. 
Table 2. Muscle blood flow during wakefulness and sleep in older piglets*

\begin{tabular}{lccc}
\hline & Wakefulness & Active sleep & Quiet sleep \\
\hline RV & $199 \pm 30$ & $190 \pm 16$ & $165 \pm 22 \dagger$ \\
LV & $238 \pm 36$ & $212 \pm 36 \dagger$ & $161 \pm 22 \ddagger$ \\
Diaphragm & $43 \pm 9$ & $29 \pm 10 \dagger$ & $24 \pm 8 \dagger$ \\
Intercostals & $21 \pm 11$ & $10 \pm 3 \dagger$ & $9 \pm 2 \dagger$ \\
Skeletal muscles & $18 \pm 8$ & $16 \pm 7$ & $13 \pm 7$ \\
\hline
\end{tabular}

${ }^{*}$ Results expressed in $\mathrm{mL} / \mathrm{min} \times 100 \mathrm{~g}$. Values are mean $\pm \mathrm{SD}$. RV, right ventricle; $L V$, left ventricle.

$\dagger p<0.05$, sleep $v$ s wakefulness.

$\ddagger p<0.01$, sleep vs wakefulness.

observed in the regional blood flows with sleep. We measured cardiac output in different animals than those already studied for blood flow determination because we wanted to avoid volume overload secondary to administration of large volumes of fluid (needed for the injections of both the Cardiogreen and microspheres). We found no significant difference in mean cardiac output between the different behavioral states at either age. Mean cardiac output was, respectively, $0.92 \pm 0.08$ and $0.90 \pm 0.07 \mathrm{~L} /$ min during wakefulness and active sleep in the young and 1.73 $\pm 0.17,1.65 \pm 0.14$, and $1.58 \pm 0.11 \mathrm{~L} / \mathrm{min}$ during wakefulness, active sleep, and quiet sleep in the older piglets.

As for the mean blood pressure, it did not change significantly in the young piglets between wakefulness and active sleep $(71 \pm$ 5 versus $69 \pm 11 \mathrm{~mm} \mathrm{Hg}$ ). In the older piglets, however, although the mean blood pressure in quiet sleep and wakefulness was not significantly different $(85 \pm 9$ versus $84 \pm 10 \mathrm{~mm} \mathrm{Hg})$, the value in active sleep ( $73 \pm 8 \mathrm{~mm} \mathrm{Hg}$ ) was significantly lower than that in the two other states.

Heart rate did not change significantly as a function of state at either age. In addition, there was no significant difference in $\mathrm{pH}$, arterial $\mathrm{CO}_{2}$ tension, or arterial $\mathrm{O}_{2}$ tension between the different behavioral states at any age. The hematocrits in the young were not different from those found in the older piglets. Finally, no significant differences were found between hematocrits at the beginning and end of the studies.

\section{DISCUSSION}

Our studies demonstrate that in piglets, behavioral state has a major influence on regional blood flow to different organ vascular beds.

Brain blood flow. The two sleep states, as compared with wakefulness, elicited sharply opposite effects in terms of blood flow to the thalamus-hypothalamus and brainstem. In fact, these regions received significantly more blood flow during active sleep and significantly less during quiet sleep than during wakefulness. The preferential distribution of blood flow to the thalamushypothalamus and brainstem during active sleep was seen at both ages, even in animals that had a lower total brain blood flow in active sleep than in wakefulness. Our results in piglets are similar to those of others in adult animals (3-5). Indeed, in their investigation of cats, Reivich et al. (3) found a preferential distribution of blood flow to the thalamus and some regions of the brainstem in active sleep as compared with wakefulness. Because of the absence of change in total brain blood flow with active sleep in our animals, this consistently higher blood flow to the thalamus-hypothalamus and brainstem probably reflects an increase in activity in these regions during active sleep. Studies in adult animals have shown an increase in neuronal activity in various regions of the brainstem and in some thalamic nuclei during active sleep as compared with wakefulness $(16,17)$. The increase in neuronal activity in the brainstem might well be related to the fact that active sleep is generated in that region of the brain and, more specifically, in the pontine reticular formation (17).

There was also no change in total brain blood flow with quiet sleep in our animals. A decrease in metabolic activity in the brainstem and the thalamus in quiet sleep is a possible explanation for the decrease in blood flow to these regions in our older piglets. Ramm and Frost (18) and Kennedy et al. (19) have shown such a decrease in metabolic activity in some regions of the brainstem and in the thalamus during quiet sleep in rats and monkeys. These authors have speculated that the decrease in metabolic activity in the thalamus during quiet sleep (an area that is concerned with the relaying of sensory information) may actually reflect functional deafferentiation of the brain during quiet sleep.

The changes in the total brain blood flow found in our study were not consistent between animals of different ages during active sleep. For example, although six of the seven young piglets had a higher blood flow in active sleep as compared with wakefulness, only about half (six of 13) of the older piglets had a higher blood flow during active sleep; three actually had a lower blood flow during active sleep than during wakefulness. This finding contrasts with previous observations demonstrating that, in both adult animals $(3,4)$ and humans $(5)$, brain blood flow is higher during active sleep than during wakefulness. The fact that active sleep is not a homogeneous state may be the main reason for the difference between our studies and those previously published. For instance, there is some evidence that changes in cerebral blood flow with active sleep depend on whether the animal is in tonic or phasic active sleep. Risberg et al. (20), for example, found a positive correlation between the frequency of rapid eye movements (characteristic of the phasic stage) and the changes in cerebral blood volume (estimate of cerebral blood flow) in adult humans; they also found that cerebral blood volume was either unchanged or lower in tonic active sleep than in the wakeful state. As well, Townsend et al. (5) found a positive correlation between cerebral blood flow and eye movement density.

In our studies, we did not attempt to separate tonic from phasic active sleep; nevertheless, it is possible that our blood flow measurements in the young piglets were determined mostly in phasic active sleep because of the predominance of phasic active sleep in newborns (21). Thus, this factor could explain why a greater proportion of young piglets had higher rates of blood flow during active sleep than during wakefulness.

The differences between our results and those of others might also be explained by the sleep deprivation factor. It is well known that sleep deprivation leads to the appearance of "rebound" active sleep, a pattern characterized by an increase in phasic events and blood flow (22). Interestingly, the cats studied by Reivich et al. (3) and the goats studied by Santiago et al. (4) were all sleep deprived; our piglets, in contrast, were not.

Muscle blood flow. The behavioral state also seems to be important in determining the blood flow to cardiorespiratory muscles. In our study, the blood flow to the diaphragm was consistently lower in active sleep than in wakefulness (over $25 \%$ ) at both ages; blood flow was also lower in quiet sleep as compared with wakefulness in the older group. We therefore raise the question as to whether the diaphragm needs less $\mathrm{O}_{2}$ and nutrients during sleep than during wakefulness. It is difficult, however to establish a functional parallel between blood flow to the diaphragm and diaphragmatic work output, inasmuch as the diaphragm work load has not been previously well assessed during sleep and wakefulness in mature and immature subjects. Ventilation, for example, is clearly not a good correlate for the work of breathing because it depends on the strength of central output and also on respiratory mechanics. Electromyographic variables, however, to the extent that they reflect work load, show that whereas phasic diaphragmatic activity in active sleep is similar to that found in wakefulness in newborns and adults (23-25), tonic activity is decreased in active sleep $(23,26)$. Therefore, if the diaphragmatic activity that occurs during sleep is not very different from that occurring during wakefulness, our blood flow results would suggest that diaphragmatic tissue extraction of $\mathrm{O}_{2}$ 
and nutrients is greater during active sleep than during wakefulness.

Other organs. The behavioral states did not influence the regional blood flow to most abdominal organs in our piglets at either age. Interestingly, however, at both ages the adrenal blood flow was significantly lower in active sleep than in wakefulness. This finding seems to correlate with the general decrease in sympathetic nervous system activity recorded in cardiac, renal, splanchnic, and sympathetic nerves with active sleep (1).

Effect of active sleep during maturation. We did not find any significant effect of age on the regional distribution of blood flow between wakefulness and active sleep in our piglets. Because our results for the brain are somewhat similar to those generally found in adult species (3-5), it seems that the effect of active sleep on regional brain blood flow is not age dependent.

It is impossible, however, for us to draw any conclusion with regard to the effect of maturation on organ blood flow (other than the brain) during sleep, inasmuch as no similar data is available in the literature for adult species. We did not, however, see any age effect in our piglets.

In summary, we have shown that although there is little difference in cardiac output and blood gas tensions between wakefulness and sleep in piglets, the behavioral states influence regional distribution of blood flow in early life in a major way, especially for the brain and skeletal muscles. Indeed, we have shown that, with respect to blood flow, the different behavioral states generate three kinds of vascular bed responses: $l$ ) an increased blood flow to some brain regions (thalamus-hypothalamus and brainstem) during active sleep and a decreased blood flow to the same regions during quiet sleep, 2) a decrease in blood flow to most skeletal muscle groups with sleep, and 3) a nonresponse in the other vascular beds to change in behavioral states. We speculate that the effect of behavioral state on brain and muscle blood flow reflects a change in local metabolic needs induced by or accompanying sleep.

Acknowledgments. The authors thank Dr. Robert B. Mellins for his continuous encouragement and the technical team of the sleep physiology laboratory.

\section{REFERENCES}

1. Coote JH 1982 Respiratory and circulatory control during sleep. J Exp Biol $100: 223-244$

2. Mancia G, Bacelli G, Adams DB, Zanchetti A 1971 Vasomotor regulation during sleep in the cat. Am J Physiol 220:1086-1093

3. Reivich M, Isaacs G, Evarts E, Kety S 1968 The effect of slow wave sleep and REM sleep on regional cerebral blood flow in cats. J Neurochem 15:301306
4. Santiago TV, Guerra E, Neubauer JA, Edelman NH 1984 Correlation between ventilation and brain blood flow during sleep. J Clin Invest 73:497-506

5. Townsend RE, Prinz PM, Obrist WD 1973 Human cerebral blood flow during sleep and waking. J Appl Physiol 35:620-625

6. Fewell JE, Williams BJ, Hill DE 1985 Control of blood pressure during sleep in lambs. Sleep 8:254-260

7. Sibley YDL, Walker AM, Maloney JE 1982 The influence of behavioural state on the cardiovascular system of newborn lambs. J Dev Physiol 4:107-119

8. Buckley NM 1983 Regional circulatory function in the perinatal period. In: Gootman N, Gootman PM (eds) Perinatal Cardiovascular Function. Marcel Dekker, New York, pp 227-264

9. Dickerson JWT, Dobbing J 1967 Prenatal and postnatal growth and development of the central nervous system of the pig. Proc R Soc Lond [Biol]

10. Curtis SE 1970 Environmental-thermoregulatory interactions and neonatal pig survival. J Anim Sci 31:576-587

11. Stanton HC, Mueller RG 1977 Performance of swine chilled during artificial rearing. Am J Vet Res 38:1003-1006

12. Heymann MA, Payne BD, Hoffman JIE, Rudolph AM 1977 Blood flow measurements with radionuclide-labelled particles. Prog Cardiovasc Dis 20:55-79

13. Schosser R, Arfors KE, Messmer K 1979 MIC-II, a program for the determination of cardiac output, arterio-venous shunt and regional blood flow using the radioactive microsphere method. Comput Programs Biomed 9:19-38

14. Haddad GG, Gandhi MR, Mellins RB 1982 Maturation of ventilatory response to hypoxia in puppies during sleep. $J$ Appl Physiol 52:309-314

15. Wallenstein S, Zucker CL, Fleiss JL 1980 Some statistical methods in circulation research. Circ Res 47:1-9

16. McGinty DJ, Beahm EK 1984 Neurobiology of sleep. In: Saunders NA, Sullivan CE (eds) Sleep and Breathing. Marcel Dekker, New York, pp 1-89

17. Steriade M, Hobson JA 1976 Neuronal activity during the sleep waking cycle. In: Kerbut GA, Phillis JW (eds) Progress in Neurobiology, Vol 6, part 3, Pergamon Press, Oxford, pp 155-376

18. Ramm P, Frost BJ 1984 Regional metabolic activity in the rat brain during sleep-wake activity. Sleep 6:196-216

19. Kennedy C, Gillin JC, Mendelson W, Suda S, Miyaoka M, Ito M, Nakamura RK, Storch Fl, Pettigrew K, Mishkin M, Sokoloff L 1982 Local cerebral glucose utilisation in non-rapid eye movement sleep. Nature 297:325-327

20. Risberg J, Gustavsson L, Ingvar DH 1969 Regional cerebral blood volume during paradoxical sleep. In: Brock M, Fieschi C, Ingvar DH, Lassen NA, Schurmann K (eds) Cerebral Blood Flow: Clinical and Experimental Results. Springer-Verlag, New York, pp 101-103

21. Roffwarg HP, Muzio JN, Dement WC 1966 Ontegenic development of the human sleep-dream cycle. Science 152:604-619

22. Dement WC, Henry P, Cohen H, Ferguson J 1967 Studies on the effect of REM deprivation in human and adult animals. In: Kety $S$, Evarts $E$, William $\mathrm{H}$ (eds) Sleep and Altered States of Consciousness. Williams and Wilkins, Baltimore, pp 456-468

23. Gothe B, Bruce EN, Goldman MD 1984 Influence of sleep state on respiratory muscle function. In: Saunders NA, Sullivan CE (eds) Sleep and Breathing. Marcel Dekker, New York, pp 241-282

24. Parmeggiani PL, Sabattini L 1972 Electromyographic aspects of postural, respiratory and thermoregulatory mechanisms in sleeping cats. Electroencephalogr Clin Neurophysiol 33:1-13

25. Tusiewiez KH, Moldofsky H, Bryan AC, Bryan MH 1977 Mechanics of the rib cage and diaphragm during sleep. J Appl Physiol 43:600-602

26. Muller N, Volgyesi G, Becker L, Bryan MH, Bryan AC 1979 Diaphragmatic muscle tone. J Appl Physiol 47:279-284 